UNIVERSITA' DEGLI STUDI DI TRENTO - DIPARTIMENTO DI ECONOMIA

\title{
THE UNREASONABLE INEFFECTIVENESS OF MATHEMATICS IN ECONOMICS
}

\author{
K. Vela Velupillai
}

Discussion Paper No. 6, 2004 
The Discussion Paper series provides a means for circulating preliminary research results by staff of or visitors to the Department. Its purpose is to stimulate discussion prior to the publication of papers.

Requests for copies of Discussion Papers and address changes should be sent to:

Dott. Edoardo Gaffeo

Dipartimento di Economia

Università degli Studi

Via Inama 5

38100 TRENTO ITALY 


\title{
The Unreasonable Ineffectiveness of Mathematics in Economics*
}

\author{
K. Vela Velupillai \\ Deaprtment of Economics \\ National University of Ireland, Galway \\ Glaway, Ireland \\ and \\ Department of Economics \\ University of Trento \\ Trento, Italy
}

September, 2003

Revised: 12 July 2004

\footnotetext{
* An earlier version of this paper was presented at the CJE Economics for the Future conference held at Cambridge University in September, 2003. Stimulating comments and constructive criticisms made at the presentation and by an anonymous referee helped reduce ambiguities and some of the harsher, critical, tones in the original version. I am, in particular, indebted to Professor Deidre McCloskey for her extremely perceptive comments on existence problems as framed and presented in the earlier version. I am also indebted to general supportive reactions and clarifying comments from Tom Boylan, Francisco Doria, Duncan Foley and Marcelo Tsuji. Alas, the remaining errors and ambiguities are entirely my own responsibility.
} 


\begin{abstract}
In this paper I attempt to show that mathematical economics is unreasonably ineffective. Unreasonable, because the mathematical assumptions are economically unwarranted; ineffective because the mathematical formalizations imply non-constructive and uncomputable structures. A reasonable and effective mathematization of economics entails Diophantine formalisms. These come with natural undecidabilities and uncomputabilites. In the face of this, I conjecture that an economics for the future will be freer to explore experimental methodologies underpinned by alternative mathematical structures. The whole discussion is framed within the context of the celebrated Wignerian theme: The Unreasonable Effectiveness of Mathematics in the Natural Sciences.
\end{abstract}

Key Words: General Equilibrium Theory, Computable General Equilibrium, Computable Economics, Constructive Mathematics, Mathematical Economics.

JEL Classification Codes: B41,C62,C68,D58 


\section{Preamble}

"Well, you know or don't you kennet or haven't I told you every telling has a taling and that's the he and the she of it."

James Joyce: Finnegan's Wake, p.213

Eugene Wigner's Richard Courant Lecture in the Mathematical Sciences, delivered at New York University on May 11, 1959, was titled, picturesquely and, perhaps, with intentional impishness The Unreasonable Effectiveness of Mathematics in the Natural Sciences, (Wigner, 1960). Twenty years later, another distinguished scientist, Richard W. Hamming, gave an invited lecture to the Northern California Section of the Mathematical Association of America with the slightly truncated title The Unreasonable Effectiveness of Mathematics, (Hamming, 1980). A decade or so later Stefan Burr tried a different variant of Wigner's title by organising a short course on The Unreasonable Effectiveness of Number Theory, (Burr, 1993). Another decade elapsed before Arthur Lesk, a distinguished molecular biologist at Cambridge, gave a lecture at the Isaac Newton Institute for Mathematical Sciences at Cambridge University where yet another twist to the Wigner theme was added: The Unreasonable Effectiveness of Mathematics in Molecular Biology, (Lesk, 2000). First a physicist; then a computer scientist; then number theorists and, finally, also molecular biologists; so why not an economist, too? But note that my title is not about the unreasonable effectiveness of mathematics in economics; I am, instead, referring to its ineffectiveness. I was not a little influenced by the story behind Arthur Lesk's eventual choice of title (Lesk, 2001).

I.M. Gelfand, a noted mathematician, had suggested as a counterpoint to Wigner's thesis his own principle on The Unreasonable Ineffectiveness of Mathematics in the Biological Sciences. Lesk, unaware of the Wigner-Gelfand principle at the time his talk was conceived, had himself suggested a similar title for his own talk at the Newton Institute but was persuaded by the organisers to retain the Wignerian flavour by dropping ineffective in favour of effective. To his surprise, when his talk was published in The Mathematical Intelligencer, the editors of the Journal, without his approval or knowledge, had inserted an inset (Lesk, 2000, p.29) describing the anecdote of the genesis of the Wigner-Gelfand principle. This prompted Lesk to recount the genesis of his own title in a subsequent issue of the Journal (Lesk, 2001) where he admitted that his preferred choice had been a title with the word Ineffective. He had proposed, to the organisers of a conference on 'Biomolecular Function and Evolution in the Context of the Genome Project', at the Newton Institute, in 1998, a talk with the title On the Unreasonable Ineffectiveness of Mathematics in Molecular Biology, which he - Lesk - thought reflected 'an echo of E.P.Wigner'. At this point the following reactions by the convener ensued:

"A prolonged and uneasy silence. Then: "But, you see, this is not quite the message that we want to send these people." More silence. Then: "Would you consider changing 'ineffective' to 'effective'?" Lesk, 2001, p.14.

Lesk acquiesced, but did go on to point out that:

"Of course, the change in title had absolutely no effect on my remarks." ibid.

There was a substantive point Lesk was trying to make with the intended title where Ineffective was emphasised in a Wignerian context. Lesk had felt that:

“...biology lacks the magnificent compression of the physical sciences, where a small number of basic principles allow quantitative prediction of many observations to high precision. A biologist 
confronted with a large body of inexplicable observations does not have faith that discovering the correct mathematical structure will make sense of everything by exposing the hidden underlying regularities.

.... A famous physicist once dismissed my work, saying: 'You're not doing science, you're just doing archaeology!' ... [I]t emphasizes a genuine and severe obstacle to applications of mathematics in biology."

ibid.

It is one of the illusions, enthusiastically maintained by mathematically competent economists, that economics is capable of a similar 'magnificent compression' of its principles to 'a small number of basic principles' that has led to the persistence faith in the application of the mathematical method in economics. Keynes famously thought if economists could manage to get themselves thought of as humble, competent people, on a level with dentists, that would be splendid' (Keynes, 1930, p.373). I would happily settle for economics being compared to archaeology and our scientific activity placed on a level with that of the archaeologist. It would be a noble analogy. As a famous mathematician who also made interesting contributions to analytical economics (Schwartz, 1961) - observed that the veneer of mathematics tends:

"[T]o dress scientific brilliancies and scientific absurdities alike in the impressive uniform of formulae and theorems. Unfortunately however, an absurdity in uniform is far more persuasive than an absurdity unclad."

Schwartz, 1986, p.22.

The aim I have set forth for myself, in this essay, is to unclothe some of the uniforms of this empress, in her economic incarnations as a mathematical economist, and show her naked torso for what it is: ineffective and non-constructive in the strict technical sense of formal recursion theory and constructive mathematics; but also to try to unclothe a few of her generals and foot-soldiers and show them in their splendid, unclad, absurdities. Others have unclothed other elements of mathematical economics in related ways and it is also possible to tell a story in terms of Unreasonably Undecidable and Incomplete economic theories ${ }^{1}$. In particular, the impressive works by what I may call the Brazilian School of Newton Costa, Francisco Doria and Marcelo Tsuji (da Costa and Doria, 1994, Tsuji, da Costa and Doria, 1998), a lifetime research program by Alain Lewis (aborted for various inexplicable reasons) and also recent work by Kislaya Prasad (2002) where, especially, the kind of questions I raise in this paper are raised in the context of game theory and its underpinnings in aspects of Nash equilibria. ${ }^{2}$ However, to keep the narrative within a concise scheme, I have had to be narrowly focused on a selected set of issues in this paper and, hence, have not harnessed the interesting results in works by these and other authors for the purpose of buttressing this story.

Wigner's essay was admirably concise (only 16 pages long) and dealt with a host of vast and deep issues within that brief number of pages. It was divided into five subsections, in addition to a brief introduction. ${ }^{3}$ I shall, to some extent, mimic that structure. Hence, the next section in this essay will try to summarise the salient points underlying alternative mathematical traditions. Wigner's brilliant lecture was delivered at a time when real analysis reigned supreme and formalism of one variety or another ruled, implicitly or explicitly. ${ }^{4}$

There was, if not universal agreement, blissful ignorance of alternative traditions that may have provided different perspectives on the formalized natural sciences. Hence, Wigner could happily confine his discussions on 'What is Mathematics?' to just a page and a half! $!^{5}$ Today such conciseness is almost impossible, even from the point of view of the knowledge of the mathematically minded economist. Classical real analysis is only one of at least four mathematical traditions within which economic questions can be formalized and discussed mathematically. Non-standard, constructive and computable analyses have been playing their own roles in the formalization and mathematization of economic entities - but mostly within the closure of neoclassical economic theory. 
Wigner's discussion of Physics and Physical theories are predicated upon the explicit and implicit fact that such theories have organising and disciplining criteria such as invariance, symmetry and conservation principles (cf. also Wigner, 1964 and McCauley, 2004). Lesk, on the other hand, by confining his discussion to that part of Molecular Biology which has come to be called Computational Molecular Biology, was able to single out the restraining and guiding hands provided by the laws of physics and chemistry, without subscribing to any kind of reductionism. He coupled these underpinnings to the mechanism of evolution and the role of chance in the latter, in particular, as organising principles to demonstrate the effectivity of mathematical theorising in Computational Molecular Biology. These organising principles operate, of course, also in Molecular Biology in general; it is just that, by concentrating on the computational subset, Lesk was able to characterize the canonical mathematical methods used as being sequence alignment and structure superposition.

If I was to follow Lesk's strategy, then I have one of four possibilities. I can either work within the framework of General Equilibrium Theory (GET) as the core of neoclassical economics and choose its computational 'subset', i.e., Computable General Equilibrium theory (CGE) and discuss the unreasonable effectiveness, or not, of mathematics inside these, narrow but well-defined citadels of application of mathematics in economics. The second possibility is to choose the computable subset of either GET or some other part of economic theory, not necessarily neoclassical in spirit, and highlight the effectivity of mathematical theorising in these subsets. The third alternative is to confine my attention to that amorphous practice, increasingly called Computational Economics, and discuss the effectivity of mathematical theorising in this field. I rule out the latter two alternatives in view of a lack of clearly defined disciplining criteria that would make it possible to provide a decent discussion within the confines of a single essay. The fourth alternative, that which appeals most to me, would be to work within the field of what I have come to call Computable Economics, but that would mean a discussion of the effectiveness of mathematics in economics, a story I have told elsewhere in greater detail (Velupillai, 2000). Therefore, I choose, in \$3, to define the 'economic theory' to which mathematics has been applied ineffectively, and unreasonably so, as GET and confine myself to brief remarks on other, related areas of economics aspiring to the status of a mathematical discipline.

I try, in $\S 4$ to suggest that we return to the tradition of the methodologies and epistemologies of the natural historian - perhaps, implicitly, also that of the dentist and the archaeologist. This final section is also a reflection of the way mathematics might develop and to speculate that the possible scenarios would reinforce the return of economics to what it once was: Political Arithmetic and to the fold of the enlightened national accountant.

\section{2. 'For Poetry Makes Nothing Happen.....' - Mathematical Traditions.}

\footnotetext{
"Among the abstract arts music stands out by its precise and complex articulation, subject to a grammar of its own. In profundity and scope it may compare with pure mathematics. Moreover, both of these testify to the same paradox: namely that man can hold important discourse about nothing." Michael Polanyi: Personal Knowledge (Polanyi, 1958, p.193; italics added).
}

If 'Poetry Makes Nothing Happen', what, then, of philosophy and mathematics? Do they make anything happen? Surely, for them - and for poetry - to make anything happen, they have to be about something. What are they about, then? Michael Dummett's enlightened and informed criteria may offer a starting point ${ }^{7}$ : 
"The two most abstract of the intellectual disciplines, philosophy and mathematics, give rise to the same perplexity: what are they about? .....

An uninformative answer could be given by listing various types of mathematical object and mathematical structure: mathematicians study the properties of natural numbers, real numbers, ordinal numbers, groups, topological spaces, differential manifolds, lattices and the like. .....

A brilliant answer to our question .. was, essentially, that mathematics is not about anything in particular: it consists, rather, of the systematic construction of complex deductive arguments. Deductive reasoning is capable of eliciting, from comparatively meagre premisses and by routes far from immediately obvious, a wealth of often surprising consequences; in mathematics such routes are explored and the means of deriving those consequences are stored for future use in the form of propositions. Mathematical theorems, on this account, embody deductive subroutines which, once discovered, can be repeatedly used in a variety of contexts."

Dummett, 1994, pp.11-14; bold emphasis added.

In other words, mathematics is about proof. I believe this to be a valid and standard characterisation which helps delineate the different 'schools' of mathematics in terms of it. Some 'routes' for the 'construction of complex deductive arguments' are aesthetically more acceptable, on clearly defined criteria, to one class of mathematicians and others to another class and this is one of the ways these different 'schools' have tried to distinguish themselves from each other. As one may expect, different 'routes' may lead the traveller to different destinations - to different classes of mathematical objects and, equally, different classes of mathematicians have approved - and disapproved, as the case may be - for aesthetic and epistemological reasons, as valid or invalid, alternative structures of 'deductive arguments'. In other words, there is no such thing as universally valid and acceptable class of 'deductive arguments' that must exclusively be used in the exploratory journeys along 'far from immediately obvious routes'. Many times, the 'routes' are discovered; at other times, they are invented. A whole, respectable and resilient, mathematical movement, methodologically and epistemologically rigorous in its ways, has always claimed that there are no 'routes' out there, laid out by the Gods, for mathematicians to discover. Mathematicians, equipped with a stock of ideas, explore alternative 'routes' with aesthetically and epistemologically acceptable deductive structures - i.e., construction rules - and create - i.e., invent - new pathways that lead to unexpected destinations. Others live in a world of Platonic shadows and discover routes that have been laid out by the Gods. ${ }^{8}$ The former are called the Intuitionists; the latter are the formal Platonists. These two classes do not, of course, exhaust the class of mathematicians; there are varieties of Platonists and, equally, varieties of Intuitionists, and others besides: Hilbertian Formalists, Bourbakists, Bishop-style Constructivists, Logicists, and so on. A flavour of the main differences, based on the Dummett-Hardy characterisation of mathematics and the mathematician, can be discerned from the following artificial dialogue between a mythical Intuitionist (I) and an undifferentiated Formalist (ME) ${ }^{9}$ :

\section{Example 1}

ME: ' I have just proved $\exists x$ s.t A.'

I: 'Congratulations. What is it? How did you prove it?'

ME: 'It is an economic equilibrium. I assumed $\forall x \neg A$ and derived a contradiction.'

I: $\quad$ 'Oh! You mean you 'proved' $\neg \forall x \neg A$ ?'

ME: 'That's what I thought I said.'

I: 'I don't think so.'

\section{Example 2}

ME: 'I have proved A $\vee$ B.'

I: 'Excellent. Which did you prove?' 
ME: 'What?'

I: 'You said you had proved A or B and I was wondering whether you had proved A or B, or both.'

ME: 'None of them! I assumed $\neg \mathrm{A} \wedge \neg \mathrm{B}$ and derived a contradiction.'

I: $\quad$ 'Oh, you mean you proved $\neg[\neg \mathrm{A} \wedge \neg \mathrm{B}]$ ?'

ME: 'That's right. Your way of stating it is simply another way of saying the same thing.'

I: 'No - not at all.'

As a direct instance of the first example, with immediate implications for the foundations of GET, there is the case of Brouwer's original proof of his celebrated fix point theorem. He - and legions of others after him, scores of who were economists - did not prove that every $f$ (in a certain class of functions) has a fixed point' (i.e., $\exists x$ s.t $A$ ). What he did prove was: 'There is no $f$ (in a certain class of functions) without a fixed point' (i.e., $\neg \forall x \neg A$ ). The equivalence between the two propositions entails an acceptance of the deductive validity of: $\neg(\neg \mathrm{A}) \square \mathrm{A}$. Brouwer himself came to reject the validity of this principle and, forty years after the initial publication of his famous result, reformulated the proof without reliance on it (Brouwer, 1952).

The second example illustrates a widely used non-constructive principle, most conspicuously utilised in the 'proof' of the Bolzano-Weierstrass Theorem (Dummett, 1977, pp.10-11), which is implicitly assumed in all 'constructions' of equilibria in CGE models. The reason for some mathematicians to object to proofs of the sort in the second example is that it shows that one or the other of two specific conditions hold without specifying a means to determine which of them is valid in any specific set of circumstance. It is as if the mathematician in his journey along those characterising 'routes' comes to a fork in the pathway and is told that one or the other of the alternatives will lead to a specified destination, but is not given any further information as to which one might do so. Is she to take both, simultaneously or one after the other - even along mathematical pathways that are routinely non-finite, as, indeed, the case in the Bolzano-Weierstrass Theorem? What are the consequences of traversing an infinite path, speckled with forks, where undecidable disjunctions can paralyse progress? The classical mathematician is not troubled by such conundrums; almost all other traditions tame undecidable disjunctions at their buds. The mathematical economist and almost all applications of mathematics in economics traverse with princely unconcern for the forks, donning the proverbial blind every time such bifurcations are encountered. No wonder, then, that the subject remains entwined and entangled in numerical indeterminacies and logical undecidabilities - but this is an item for the next section.

In the above explicit instance of the first example I have invoked the idea of a function without trying to define its meaning. So, what is a function ( $^{10}$ How do different mathematical traditions confront the task of answering this question? The ordinary meaning of the word 'function' is associated with the 'idea' of performing a task. All mathematical traditions, with the notable exception of what, for want of a better name, I shall call 'classical real analysis' or 'classical mathematics', each in their own way, retain fidelity to the ordinary meaning of the word 'function' in their specialised characterisations. Historically, in mathematics, the meaning of the concept was intimately tied to the notion of a rule, a procedure, a set of instructions to perform a task. Thus, for example, a function $f$ was supposed to enable a mathematician to calculate, given a number, say $x$, - real, natural, or whatever - another number, denoted by $f(x)$ such that, whenever $x=y$, then $f(x) f(y)$. This was to impose some disciplining criteria in the procedures - the methods by which patterns are created. However, at the hands of the classical mathematicians this became ossified as the well-known Kuratowski-Dirichlet definition ${ }^{11}$ :

\section{Definition 1:}

A function $f: A \rightarrow B$ is any subset $f \subseteq(A \times B)$ which satisfies: $(\forall x \in A)(\exists y \in B)$ s.t $(x, y) \in f \&\left(x, y^{\prime}\right) \in f \Rightarrow y=y^{\prime}$. 
However, this definition - 'function as a graph' - makes sense only within set theory ${ }^{12}$. The definition has severed all connections with the meaning attributed to the word 'function' in ordinary discourse; there is little sense in which it can be understood to 'perform a task'. The idea of a 'rule', a 'procedure', encapsulated within the historical definition of the idea - concept - of a 'function' has disappeared. This is best illustrated by an example (cf. Moschovakis, 1994, p.41). The following 'formulas' for computing the square of two numbers, defined on the reals, are equivalent in the 'function as a graph' definition implied by the above Dirichlet-Kuratowski characterization:

$$
\begin{gathered}
f(x, y) \equiv(x+y)^{2} \\
g(x, y) \equiv x^{2}+2 x y+y^{2}
\end{gathered}
$$

As tasks to be performed, say on a digital computer via a simple program, they result in different sets of instructions. The key point is this: whether the notion of a function that is based on 'performing a task' can be represented in set theory in such a way as to capture its full intuitive content remains an open question. In spite of this indeterminacy, mathematical economists - and, so far as I know, all economists who apply mathematics in economics - routinely rely on this particular definition for their so-called rigorous notion of a function.

On the other hand, almost all other traditions, as mentioned above, in their definitions of the notion of a function, retain fidelity with the ordinary meaning and mathematical tradition. Thus, in Bishop-style constructive mathematics the distinguishing starting point is that all existence proofs should be constructive in the precise sense that every proof can be implemented, in principle, as an algorithm in a computer to demonstrate, by explicit construction, the object in question. This means, firstly, that the law of the excluded middle (tertium non datur) is not invoked in infinitary cases; secondly, as a by-product of such a discipline on existence as construction, all functions are required to be uniformly continuous in each closed interval. In other words, if mathematics is about proving theorems, and if proofs are to be constructive - i.e., performable tasks, at least in principle, by a set of explicit instructions - then each function must be characterized in a certain precise way. Hence, Bishop-style constructive mathematics retains fidelity with the ordinary meaning of the concept of function by endowing it with certain mathematical properties - i.e., uniformly continuous in each closed interval - such that when they are used in the pattern formation activities of the mathematician they will facilitate the 'performance of tasks'.

In that variant of constructive mathematics known as Brouwerian Intuitionism, the starting point is what is known as 'free choice sequences' - where a rule for determining a real number is a result of free choices by an autonomous human intelligence, independent of the strictures of the undecidable disjunctions of classical logic. This implied, in Brouwerian Intuitionism, that all functions from reals to reals are continuous. Here, too, starting from a metatheoretic assumption - construction of the primitives by 'free choice sequences', based on what Brouwer considered was the domain of activity of the mathematician - his or her autonomous intelligence - one was led to consider a characterisation of functions that retained fidelity with tradition and the ordinary meaning of the word.

Then, there is the class of computable functions, the domain of the recursion theorist, acting under the discipline of the Church-Turing Thesis. The most direct way of describing or characterising these functions - although not the mode that I find most congenial - is to say that they are that subset of the functions defined in classical mathematics which can be implemented on an ideal digital computer i.e., the Turing Machine. Next, invoking the Church-Turing Thesis, one identifies them, depending on the aims of the analysis, as the class of partial recursive functions or Church's $\lambda$-definable functions, etc. Then, by way of elementary counting arguments it is shown that there are 'only' a countable infinity of Turing Machines and, hence, also of partial recursive functions, implying thereby that the complement of this set in the class of all classically defined functions contains the uncomputable 
functions. They are, therefore, uncountably infinite in number! This, by the way, is the class of functions routinely used and assumed in mathematical economics of every variety, without exception.

It is, of course, possible to continue a finer classification of varieties of constructive mathematics and, also, varieties of Formalists, Platonists, and Logicists and so on ${ }^{13}$. However, this will achieve no particular purpose beyond that which has been achieved with the above few considerations and characterisations for the following reasons. Given the Hardy-Dummett characterisation of mathematics and the activity of the mathematician in terms of 'the systematic construction of complex deductive arguments', it was inevitable that there would be some dissonance in the meaning and interpretation to be attached to 'construction' and the acceptability or not of valid deductive rules for the 'construction'. Depending on the kind of deductive rules and constructions accepted as valid, there are different ways to characterise mathematics and mathematicians. I have highlighted a few of the possible ways to do this - but many other ways could have been attempted with equal ease, which would have resulted in a many-splendoured world of possible mathematics and mathematicians. The main point to note is that it is not a monolithic world, characterised by one concept of 'proof' and a single way of 'constructing patterns' from an inflexibly determined set of deductive rules.

\title{
3. A Glittering Deception of Entrappers \& Entrapped ${ }^{14}$
}

\author{
"And he wondered what the artist had intended to represent (Watt knew nothing about painting), ... a \\ circle and a centre not its centre in search of a centre and its circle respectively, in boundless space, in \\ endless time (Watt knew nothing about physics) ... ." \\ Samuel Beckett: Watt (Beckett, 1963, p.127); italics added.
}

Given the availability of a variety of mathematical structures that could have been harnessed for the formalization of economic theory, why and how did economists choose the one formalism that was most conspicuously devoid of numerical content ${ }^{15}$ ? This question, to be answered, requires a detailed historical study of source material in disciplined ways, and is, in any case, outside the particular scope of this paper. What is, however, clear beyond any doubt is the crucial role played by existence proofs in the modern mathematization of economic theory. To this may be added also the role of separating hyperplane theorems, in view of the importance of optimization as a disciplining criterion in economic analysis. I shall, therefore, for the purposes of the discussion in this paper have these two issues in mind although, paradoxically, the latter - separating hyperplane theorems - is less crucial in a formal sense than the former - fixed point theorems. At the very outset I would like to make it very clear that, contrary to widespread and popular belief that topological fixed point theorems are the only way to formalize economic equilibrium existence problems, this is demonstrably false. By 'demonstrably false' I mean the following: it is easy to formalize, constructively or recursion theoretically, the fundamentals of economic theory - rational agents and their choice behaviour - and seek equilibria using perfectly valid constructive or computable fixed point theorems. For example, at a most basic level, the problem of formalizing rational expectations, showing its existence and deriving a learning scheme to achieve it can all be framed recursion theoretically and constructively (cf. Velupillai, 2004).

In their seminal textbook on mathematical economics, Arrow and Hahn (1971) state that their 'methods of proof are in a number of instances quite different' from those in Debreu's classic, codifying, text on the Theory of Value (Debreu, 1959). Debreu, in turn, claimed that he was treating the theory of value, in his book, 'with the standards of rigor of the contemporary formalist school of mathematics' and that this 'effort toward rigor substitutes correct reasonings and results for incorrect ones' (ibid, p.viii). But we are not told, by Arrow and Hahn or by Debreu, either what these 'different methods of proof' mean in the form of new insights into economics (or mathematics) or which concept of 'rigor' underpins the substitution of 'correct reasonings and results for incorrect ones'. 
On the other hand, the crowning achievement of the Arrow-Debreu reformulation of the Walrasian problem of the existence of economic (exchange) equilibrium was its formal demonstration as the solution to a fixed point problem. In addition to this, there was the harnessing of theorems of the separating hyperplane - more generally, the Hahn-Banach Theorem and Duality Theorems - to formally demonstrate the mathematical validity of the two fundamental theorems of welfare economics. Thus, existence of economic equilibrium and welfare economics were given so-called rigorous mathematical formulations and formal demonstrations as theorems of various sorts. Both Arrow and Debreu were handsome in their acknowledgement of debts to the trails that had been blazed by the pioneers in mathematical method for such issues: von Neumann, Wald and Nash being the most prominent among them, but also numerous mathematicians - Brouwer, Kakutani and Banach, to name the obvious ones.

As a sequel to the codification achieved by Debreu, Scarf began a sustained research program to 'effectivize' and 'constructivise' one aspect of the mathematics of general equilibrium theory: the problem of existence. Early on, he had realised that proving existence by non-constructive means was unsatisfactory from the point of view of economics as an applied subject, even apart from possible aesthetic motivations and intellectual challenges to constructivise non-numerical concepts. This is the research program under the rubric of Computable General Equilibrium theory (CGE), with far reaching policy implications. Surprisingly, no one has tried to constructivise or effectivize the formalizations of the two fundamental theorems of welfare economics, on which momentous policy prescriptions - even of a philosophical nature - depend.

The main question I wish to pose in this section is the following: suppose the modern masters of mathematical general equilibrium theory had been more enlightened in their attitude and, possibly, knowledge of mathematics and its philosophy, ontology and epistemology, and had they taken the trouble to 'treat the theory of value with the standards of rigour of not only 'the contemporary formalist school of mathematics', but with the 'standards of rigour' of other contemporary schools of mathematics, how much of their economic propositions would remain valid? In other words, did the successes of the Theory of Value depend on the fortuitous fact of having been formalised in terms of 'the contemporary formalist school of mathematics'?

A subsidiary question I pose, next, is whether Scarf's program can be carried through successfully. The claim, by leading applied economists, is that it has been carried through successfully and GET is, now, an eminently applicable field, with clear computational and numerical content.

My answer to the first question is that the results are decisively sensitive to the kind of mathematics used. The answer to the second question is that the Scarf program cannot succeed in its aim to constructivise the equilibrium existence problem of GET, i.e, the constructive and computable content of CGE is vacuous.

Before I consider the unreasonable ineffectiveness of mathematical general equilibrium theory, there are a few ghosts to rekindle, a phantom to resurrect and some to lay to rest. The first ghost that deserves a rekindling is the existence problem - and from two points of view. Firstly, is it really necessary to pose, as a mathematical, problem, the question of equilibrium existence in formal economic theory? Hicks, arguably the doyen of economic theory for the 20th century, did not think so:

"[T]he [Value and Capital] model is not much affected by the criticism, made against it by some mathematical economists, that the existence of an equilibrium, at positive prices, is not demonstrated.

.... Existence, from my point of view, was a part of the hypothesis: I was asking, if such a system existed, how would it work?"

Hicks, 1983, p.374; italics added.

With an eye at some questions to be raised below, let me ask ${ }^{16}$ : why 'at positive prices' and not 'at positive integer or rational prices'? 
Next, even if there is a satisfactory answer to the first question - in spite of the weight of Hicks' vision and stand - was it necessary to formulate the equilibrium existence problem as a fix point problem? Smale did not think so:

\footnotetext{
"We return to the subject of equilibrium theory. The existence theory of the static approach is deeply rooted to the use of the mathematics of fixed point theory. Thus one step in the liberation from the static point of view would be to use a mathematics of a different kind. Furthermore, proofs of fixed point theorems traditionally use difficult ideas of algebraic topology, and this has obscured the economic phenomena underlying the existence of equilibria. Also the economic equilibrium problem presents itself most directly and with the most tradition not as a fixed point problem, but as an equation, supply equals demand. Mathematical economists have translated the problem of solving this equation into a fixed point problem.

I think it is fair to say that for the main existence problems in the theory of economic equilibrium, one can now bypass the fixed point approach and attack the equations directly to give existence of solutions, with a simpler kind of mathematics and even mathematics with dynamic and algorithmic overtones."

Smale, 1976, p.290; bold emphasis added.
}

A phantom to resurrect, in this connection, especially in the context of the forum for which this essay was prepared, is that a supreme example of equation systems that were solved without recourse to any kind of fixed point theorem, were those presented by Piero Sraffa (Sraffa, 1960). Of course, the Sraffa systems were not of the supply=demand variety; nevertheless, they were equilibrium systems of a sort. That legions of mathematical economists, both well-meaning and hostile, spent time and effort to re-prove what had been proved quite adequately, although not by formalistic means, remains an unfathomable mystery to me. It was as if no one could understand simple, constructive proofs or, worse, that even mathematically competent readers were one-dimensional in their knowledge of techniques of proofs. Why someone did not use Sraffa's perfectly adequate and competent methods to re-prove, say, the Perron-Frobenius theorem, and free it from the shackles of reliance on the nonconstructive Brouwer fixed point theorem is also a mystery to me. Kurz and Salvadori (2001) make this point, but remain, unfortunately, shackled within the prisons of real analysis and, hence, their alleged 'algorithm', however well-meaning, is unimplementable (ibid, p.206). The precise reason is, in view of Specker's Theorem in Computable Analysis (cf. Theorem 1, below), their appeal to the classic theorem of real analysis, where a bounded monotone sequence converges, is invalid in algorithmic analysis. Moreover, it is not clear from their sympathetic discussion whether they refer to algorithms in the sense of constructive or computable analysis. This is an example, also, of how entrenched traditional mathematical and logical habits are, among even well-meaning mathematical economists, and extracting the non-numerical and non-effective content of unguarded propositions is both tedious and arduous. Sraffa, in the main part of his book, did not invoke or use any non-algorithmic method of analysis in demonstrating his propositions. I developed some aspects of these themes and the attendant difficulties a long time ago (cf. for example, Velupillai, 1989 and references cited therein), but had no success in eliciting any interest, favourable or otherwise.

Why, then, did mathematical economists translate the problem of solving equations 'into a fixed point problem' of proving the existence of a solution? Also, suppose we return to the 'equation' tradition but impose natural economic constraints on the variables, parameters and constants of the supply-demand relations. Such natural constraints would imply integer and rational valued variables, constants and parameters. To return to a variant of the question I posed just after the Hicks quote: why the fetishism of looking for 'non-negative prices' in an equilibrium configuration? Surely, a return to the equation tradition, with non-negative integer or rational valued variables, constants and parameters means a confrontation with a combinatorial monster: Diophantine equation. In such an environment, the economic problem would naturally become a (recursion-theoretic) decision problem and will no longer be a traditional optimization problem. 
Let me, now, return to GET and CGE and their mathematically unreasonable ineffectiveness. Here I shall mean, by ineffectiveness, the strict technical sense of being uncomputable or non-constructive. The caveat unreasonable signifies the fact that the mathematics used - i.e., methods of proof utilized in GET and CGE - and the axioms assumed - were not only economically injudicious but also unnecessary and irrelevant from every conceivable numerical and computational point of view.

The formal underpinnings of the economic theory enunciated in Debreu's Theory of Value depend crucially on the following mathematical axiom, concepts and theorems ${ }^{17}$ :

1. The axiom of completeness ( $§ 1.5 . d, \mathrm{p} .10)$;

2. Compactness $(\S 1.6 . t, \mathrm{p} .15)$;

3. (Topologically characterized) Continuity (§1.7.b, p.15);

4. The maximum-minimum theorem or, as Debreu has named it, the Weierstrass theorem (§1.7.h (4'), p.16);

5. The separating hyperplane theorems ( $§ 1.9, \mathrm{pp} .24-5)$;

6. The Brouwer and Kakutani Fixed Point Theorems (\$1.10, p.26);

Let me, now, add, to this mathematical apparatus in the Theory of Value, the following six theorems, propositions and facts:

Theorem 1: Specker's Theorem in Computable Analysis

A sequence exists with an upper bound but without a least upper bound.

Proposition 1: The Heine-Borel Theorem (Compactness) is invalid in Computable Analysis.

Claim 1: There are 'clear intuitive notions of continuity which cannot be topologically defined' (Gandy, 1995, p.73).

Proposition 2: The Bolzano-Weierstrass Theorem is invalid in Constructive Mathematics.

Claim 2: The Hahn-Banach Theorem is invalid in its classical form in Constructive and Computable analysis.

Claim 3: The fixed point theorems in their classical versions are not valid in (Intuitionistically) Constructive Mathematics.

If the above theorem, propositions and claims are appended to the Theory of Value, or to any later 'edition' of it such as Arrow-Hahn (1971), then it can be shown that none of the propositions, theorems and claims of a mathematical sort would retain their validity without drastic modifications of their economic content and implications. In particular, not a single formal proposition in the Theory of Value would have any numerical or computational content ${ }^{18}$.

Suppose we add, to the above six supplementary 'riders', the following Claim on the Uzawa Equivalence Theorem (Uzawa, 1962):

Claim 4: The Uzawa Equivalence Theorem is neither constructively nor computably valid.

Then, in conjunction with the invalidity of the Bolzano-Weierstrass Theorem (Proposition 2, above), Claim 4 implies that the constructive content of CGE models, and their computational implications for economic policy analysis, are vacuous.

A similar exercise can be carried out for every sub-field of economic theory to which the mathematical method has been applied - in particular, game theory. It will be a tedious exercise but I suspect that, eventually, such an exegesis can even be automated! The general strategy would be to identify the key mathematical axioms, theorems and concepts that underlie any particular mathematics 
applied to a sub-field of economic theory and, then, to investigate their constructive, computable, nonstandard or real analytic nature. Thus, for example, a seemingly innocuous application of dynamical systems theory in endogenous theories of the business cycle would also be susceptible to such an exegetic exercise. Any use of the Cauchy-Peano theorem in the existence theory for differential equations will fall foul of the failure of the validity of the Bolzano-Weierstrass Theorem in Constructive mathematics. This is because the Bolzano-Weierstrass Theorem is equivalent to the Ascoli Lemma which, in turn, is used to simplify the proof of the Cauchy-Peano Theorem.

In passing, it must, of course, be pointed out that fixed point theorems did not enter economic analysis by way of the existence problem of general equilibrium theory; the entrance points were game theory and growth theory - both at the hands of von Neumann. For reasons of space, my remarks on these two issues will have to be brief. First of all, as regards game theory, I have already tried to make a case for recasting every game theoretic problem in economics as an Arithmetical Game (cf. Velupillai, 2000, ch.7). This implies that their solutions can be reduced to Diophantine decision problems, in analogy with the equation approach to the economic equilibrium existence problem. Secondly, in the case of growth theory, the original fixed point problem of a minimax system was 'simplified' into a separating hyperplane problem. But as pointed out above, the separating hyperplane theorem, or the Hahn-Banach theorem, has neither an exact equivalent formulation in constructive mathematics nor is it known, at present, whether it is valid in computable analysis (cf. for example Metakides and Nerode, 1982). However, the fact remains that growth theory is a problem of selfreproduction and self-reconstruction, and to that extent the theory can felicitously be reformulated as a recursion theoretic problem and the standard, numerically implementable, fixed point theorem of recursion theory can be applied.

What kind of lessons are we to draw from this particular exercise in exegesis? There is almost no better way to phrase the main lesson to be drawn than in the words of a leading mathematical macroeconomist:

".. [A]s economic analysts we are directed by, if not prisoners of, the mathematical tools that we possess."

Sargent, 1987, p.xix; italics added.

Should we not, if we are 'prisoners' of anything, try to liberate ourselves from that which imprisons us?

\section{4. '..The Path We Will Never Walk Again'19}

\footnotetext{
"Mathematics is not a finished object based on some axioms. It evolves genetically. This has not yet quite come to conscious realization....

[T] here might someday be entirely new points of view, even about sets or classes of sets. Sets may someday be considered as 'imaginary.' I think that will come to pass, though at present it is not admissible.

Mathematics will change. Instead of precise theorems, of which there are now millions, we will have, fifty years from now, general theories and vague guidelines, and the individual proofs will be worked out by graduate students or by computers.

Mathematicians fool themselves when they think that the purpose of mathematics is to prove theorems, without regard to the broader impact of mathematical results. Isn't it strange?

In the next fifty years there will be, if not axioms, at least agreements among mathematicians about assumptions of new freedoms of constructions, of thoughts. Given an undecidable proposition, there will be a preference as to whether one should assume it to be true or false. Iterated this becomes: some statements may be undecidably undecidable. This has great philosophical interest."

Ulam (Cooper, 1989, pp. 310-2); italics added.
} 
Ulam was always a prescient mathematician. In an almost uncannily eerie confirmation of his broad prediction, Stephen Wolfram's much heralded, widely discussed, somewhat controversial New Kind of Science (Wolfram, 2002; henceforth, NKS) has set out an implementable version and vision of the kind of mathematics that may have been in Ulam's mind ${ }^{20}$. It is also appropriate because Wolfram's instrument to implement the vision is a particular construction which Ulam (together with von Neumann) pioneered: the Cellular Automaton. Wolfram's broad philosophical stance on the nature and evolution of mathematics buttresses my own vision and, in many ways, is a variant of Ulam's as I have (admittedly selectively) summarised above:

"...like most other fields of human enquiry mathematics has tended to define itself to be concerned with just those questions that its methods can successfully address. And since the main methods traditionally used in mathematics have revolved around doing proofs, questions that involve undecidability and unprovability have inevitably been avoided. ....

The main point .. is that in both the systems it studies and the questions it asks mathematics is much more a product of its history than is realized."

ibid, p.792.

If economics is formalized using traditional mathematics, then, naturally, it will be crippled or enhanced by the poverty or richness of the history that determines the latter, as the natural sciences have been, a point emphasised by Ulam and Wolfram; but also, the extent to which 'mathematics has tended to define itself to be concerned with just those questions that its methods can successfully address', will distort the economic theory that is formalized using that particular kind of mathematics. I have tried, within the limited span of the previous sections, to make clearer than is traditionally done in mathematical economics, the traditionally invoked methods of mathematics and their limitations (and, to some extent their historically evolved nature). Much more can, of course, can be said and discussed about these aspects but it is satisfying to find my interpretations echoed in the stances taken by Ulam and Wolfram.

The place and kind of mathematical principles and practice I envisage, in an economics for the future, outlined below and, partly, implicit in the way ineffectivity of standard mathematical economics was discussed above, is such that it will be perfectly sensible and practicable to consider it a particular implementation of the message in $N K S$, especially as developed in chapter 11: The Notion of Computation and chapter 12: The Principle of Computational Equivalence (ibid).

If I might try to compress the essential methodological message in $N K S$, for the purpose of a brief justification of the feasibility of the embedding mentioned in the footnote above, then it would be something like the following. On the basis of a mathematical philosophy of the formal meaning of computation, underpinned by recursion theory, Wolfram fundamental ontological assumption, in an almost (early) Wittgensteinian way, is that everything is to be viewed as a process or as the realization of a process and (ibid, p.715):

\section{All processes can be viewed as computations}

With this as his basic epistemological vision, he enunciates the basic principle of $N K S$ to be $\underline{T h e}$ Principle of Computational Equivalence (PCE), (ibid, pp. 716-7):

\section{Almost all processes that are not obviously simple can be viewed as computations of equivalent structure}

To give methodological content to the idea of computations of equivalent structure in this principle he invokes the notion of computation universality, borrowed from recursion theory, suitably 
modified, but leaves the notion of 'not obviously simple' incompletely formalized, but not hanging by the proverbial bootstraps. From PCE he derives, as a consequence, what is best viewed as a Phenomenological Law, such as those commonly enunciated in phenomenological thermodynamics: Computational Irreducibility.

The above, in a nutshell, summarizes the ontology, epistemology and methodology underpinning $N K S$. Essentially, computational irreducibility of a process, by $P C E$, implies unpredictability. $\mathrm{He}$ is then able to show that traditional science is, basically, computationally reducible and, hence, can succeed in local predictability. This is because the mathematical laws that encapsulate traditional science are simple enough to be analytically solvable and, hence, are computationally reducible.

In other words, he extracts the implicit processes intrinsic to or implied by any mathematical law or formalism and uses his $P C E$, and its phenomenological consequence, computational irreducibility to evaluate their effective and predictable content to show the simplistic, unrealistic and undesirable nature of mathematical formalisms in traditional science. However, his message can be read as a way of showing the unreasonable effectiveness of mathematics in traditional science. This is because, his aim is to show that the appearance of the success of traditional science, using the criteria of simplicity in the laws that encapsulate the phenomena to be explained and the predictions that are extracted from them, is due to a subterfuge: that of concentrating on using analytically solvable mathematical formalisms to encapsulate natural laws and thereby ignoring the processes that have to be used to evaluate the solution to any such formalism. In this way, the methodology of traditional science circumvented the implications of computational irreducibility and, hence, ignored PCE.

The exercise I have attempted in the previous sections is very similar to Wolfram's broader agenda and can be embedded within his scheme - if I were to accept his ontology. I am not entirely sure I can, at this juncture, subscribe to it or to its underpinnings. I do not confine myself to algorithms or computations in a strictly recursion theoretic way; I have left the door open to constructive mathematics and, hence, to algorithms - computational processes - that are not subject to the ChurchTuring thesis and to the possibility of relying on intuitionistic logic. However, I am nor unhappy not unwilling to embrace his methodology and epistemology; indeed, I have done so quite willingly in my other writings, except that I base myself on a minor variant of his computational irreducibility, known in algorithmic complexity theory as incompressibility. Further excursions into these issues will complicate and expand this paper and its message beyond reason and space and I must leave it for a different exercise (cf. Albin, Foley and Velupillai, 2004).

To return, now, to the themes of the previous section it is surely true that, more than any other single factor, it is the obsession with the existence problem that seems to have condemned mathematical economics to ineffectivity. But this is not as serious a problem for the mathematization of economic theory in an effective mode as it might appear at first sight. Though it may require hard work - in the sense of having to re-learn alternative mathematical paradigms - it is not, intrinsically an impossible task. Nor will the knowledge and traditions gained in training ourselves to think formally and formalise routinely go to waste. It is almost a routine task to formalize economic theory recursion theoretically or constructively and to highlight its natural quantitative underpinnings explicitly.

On the other hand, it is not for nothing that the great master of modern economic theory, sometimes even in its mathematical mode, John Hicks, never tired of emphasising the importance of the accounting tradition in economic analysis, particularly dynamic economics:

"In all its main forms, modern economic dynamics is an accounting theory. It borrows its leading concepts from the work which had previously been done by accountants (with singularly little help from economists); and it is in accordance with this that social accounting should be its main practical instrument of application."

Hicks, 1956, p. 221. 
Somewhere between the Political Arithmetician, alias the National Income Accountant, and the Financial Analyst, alias the Accountant, lies the task of the quantitative economist's analytical role and none of the theoretical or applied tasks of these two pragmatic and paradigmatic figures requires anything more than arithmetic, statistics and the rules of compound interest. These, in turn, require nothing more than an understanding of the conditions under which systems of equations can and cannot be solved. But what kind of quantities do these equations encapsulate as parameters, constants and variables? ${ }^{21}$ Surely, the kind of quantities that enter the equations of the Political Arithmetician and the Accountant cannot be other than rational or natural numbers - negative and non-negative? ${ }^{22}$. Eminent theorists, working in core areas of economic theory - price theory and monetary theory have made this point in interesting ways over the past half a century (Gomory and Baumol, 1960, Clower \& Howitt, 1978 and Scarf, 1990).

For example, in one of the most fundamentally innovative discussions on a core area of monetary economics, Clower and Howitt remarked that a realistic analysis of the Transactions Theory of the Demand for Money implied the use of proof techniques involving 'the use of number theory - a branch of mathematics unfamiliar to most economists' (ibid, p.452, footnote 3). The reason such proof techniques had to be used was that they imposed simple, realistic, constraints on the domain of analysis - integer units. Their starting point was that individual traders produce, sell and purchase only discrete, integer, units of one stock-flow good - the obvious and natural place to begin. Once this is done, the economic problem automatically becomes a Diophantine decision problem, although the unwary reader may not immediately recognise this aspect when they are formulated as integer programming problems (as in Scarf, op.cit). But in a classic paper on a traditional problem in economic theory, pricing, Gomory and Baumol made the connection between integer programming and Diophantine problems right at the outset:

\footnotetext{
"A surprisingly wide range of problems including Diophantine problems .... can be given an integer programming formulation."

ibid, p.521; italics added
}

Why these hints, implorations and suggestions were not taken more seriously in the practice and teaching of economic analysis, I do not know; perhaps inertia is the simplest explanation. In any case, I cannot see any role for real numbers in quantitative economics and, hence, none whatsoever for real analysis and the proof techniques allied to it. Richard Hamming wondered, similarly, about the appropriate kind of numbers for probability theory ${ }^{23}$ :

"Thus without further examination it is not completely evident that the classical real number system will prove to be appropriate to the needs of probability. Perhaps the real number system is: (1) not rich enough - see non-standard analysis; (2) just what we want - see standard mathematics; or (3) more than is needed - see constructive mathematics, and computable numbers. ...

What are all these uncountably many non-computable numbers that the conventional real number system includes?....

The intuitionists, of whom you seldom hear about in the process of getting a classical mathematical education, have long been articulate about the troubles that arise in the standard mathematics ....

What are we to think of this situation? What is the role in probability theory for these numbers which can never occur in practice?"

Hamming, 199, pp. 190-1; italics in original.

Thus, the only kind of equations that can play any role in the analytical activities of the Political Arithmetician and the Accountant are Diophantine equations. How can the problem of solvability of such equations be studied and what methods are available to systematise and routinise their use? The paradoxical answer to both of these questions is that the problem of solvability is intractable and their systematic and routinised study is almost impossible. They share, with that other 'Cinderella of pure 
mathematics', nonlinear differential and difference equations, a Linnean status, as poignantly and accurately described by George Temple ${ }^{24}$ :

"The group of problems which I propose to describe belong to that Cinderella of pure mathematics- the study of Diophantine equations. The closely guarded secret of this subject is that it has not yet attained the status and dignity of a science, but still enjoys the freedom and freshness of such pre-scientific study as natural history compared with botany. The student of Diophantine equations ... is still living at the stage where his main tasks are to collect specimens, to describe them with loving care, and to cultivate them for study under laboratory conditions. The work of classification and systematization has hardly begun.

... An inviting flora of rare equations and exotic problems lies before a botanical excursion into the Diophantine field."

Temple, 1958, p.233; italics added.

Why are they intractable? How will they relate to the more conventional analytical approaches via the behaviour of rational agents? Indeed, what kind of animals are they? I cannot, of course, go into the full details of these 'inviting flora of rare equations' but shall try to provide a glimpse into their 'closely guarded secrets' (using the formalism and definitions in Matiyasevich, 1993).

Definition 2: A relation of the form:

$D\left(a_{1}, a_{2}, \ldots . ., a_{n}, x_{1}, x_{2}, \ldots, x_{n}\right)=0$

where, $D$ is a polynomial with integer coefficients with respect to all the variables (integer or rational valued):

$\left\{a_{1}, a_{2}, \ldots . ., a_{n}, x_{1}, x_{2}, \ldots, x_{n}\right\}$

separated into parameters:

and unknowns:

$$
\left\{a_{1}, a_{2}, \ldots ., a_{n}\right\}
$$

$$
\left\{x_{1}, x_{2}, \ldots, x_{n}\right\}
$$

is called a Parametric Diophantine equation.

\section{Definition 3:}

$D$ in Definition 2 defines a set $\mathfrak{I}$ of the parameters for which there are values of the unknowns such that:

$$
\left\langle a_{1}, a_{2}, \ldots, a_{n}\right\rangle \in \mathfrak{I} \Leftrightarrow \exists x_{1}, x_{2}, \ldots, x_{m}\left[D\left(a_{1}, a_{2}, \ldots, a_{n}, x_{1}, x_{2}, \ldots, x_{m}\right)=0\right]
$$

Loosely speaking, the relations denoted in the above two definitions can be called Diophantine representations. Then sets, such as $\mathfrak{I}$, having a Diophantine representation, are called simply Diophantine. With this much terminology at hand, it is possible to state the fundamental problem of Diophantine equations as follows:

Problem: A set, say:

$$
\left\langle a_{1}, a_{2}, \ldots, a_{n}\right\rangle \in \mathfrak{J}
$$

is given. Determine if this set is Diophantine. If it is, find a Diophantine representation for it. 
Of course, the set $\mathfrak{I}$ may be so structured as to possess equivalence classes of properties, $P$ and relations, R. Then it is possible also to talk, analogously, about a Diophantine representation of a Property P or a Diophantine representation of a Relation R. For example, in the latter case we have:

$$
R\left(a_{1}, a_{2}, \ldots . ., a_{n}\right) \Leftrightarrow \exists x_{1}, x_{2}, \ldots, x_{m}\left[D\left(a_{1}, a_{2}, \ldots, a_{n}, x_{1}, x_{2}, \ldots, x_{m}\right)=0\right]
$$

Hence, given, say partially ordered preference relations, it is possible to ask whether it is Diophantine and, if so, search for a Diophantine representation for it. Next, how can we talk about the solvability of a Diophantine representation? This is where undecidability (and uncomputability) will enter this family of 'inviting flora of rare equations' - through a remarkable connection with recursion theory, summarized in the next Proposition:

\section{Proposition 3:}

Given any parametric Diophantine equation, $D$, it is possible to construct a Turing Machine, $\mathrm{M}$, such that M will eventually Halt, beginning with a representation of the parametric $n$-tuple:

$$
\left\langle a_{1}, a_{2}, \ldots, a_{n}\right\rangle
$$

iff $D$ in Definition 2 is solvable for the unknowns:

$$
\left\{x_{1}, x_{2}, \ldots, x_{n}\right\}
$$

But, then, given the famous result on the Unsolvability of the Halting problem for Turing Machines, we are forced to come to terms with the unsolvability of Diophantine equations.

\section{Remark:}

Given a parametric Diophantine equation, it is possible to use the set of values of its solution to emulate the behaviour of a suitably constructed cellular automaton. Conversely, given a universal cellular automaton, it is possible to encode any behaviour of its evolution, for given initial conditions, as a statement about the solution to a parametric Diophantine equation. The intractability of characterising the limit sets of the dynamics of cellular automata, the unsolvability of the Halting problem for Turing Machines and the undecidability of the solutions for parametric Diophantine equations are all aspects of the irrelevance of the search for axiomatic encapsulation and compression of fundamentals.

Thus, once the natural Diophantine formalism of accounting systems are recognised and routinised, their general unsolvability should lead to the kind of 'assumptions of new freedoms of constructions, of thoughts' and a shift away from 'precise theorems and axioms', as envisaged by Ulam and Wolfram. I shall go further and suggest, for an economics of the future, the notion of a Universal Accounting System, implied by and implying Universal Turing Machines and Universality in cellular automata (or dynamical systems, in general), to study value, distribution and growth.

Hence, the best we can do, as Political Arithmeticians and Accountants, and even as behavioural agents, however rational, so long as the constraints are Diophantine, is to act according to the gentle and humble precepts enunciated by George Temple: 'collect specimens, to describe them with loving care, and to cultivate them for study under laboratory conditions'. Clearly, anyone familiar with the work of Charles Sanders Peirce will also realise that this kind of natural historic study fits comfortably with that great man's advocacy of retroduction ${ }^{25}$ in such disciplines. The tiresome dichotomy between induction and deduction, refreshingly banished by Peirce more than a century ago, may well get 
cremated in economics, once and forever, if we combine the methodology of the natural historian with the epistemology that is implied in retroduction.

The headlong rush with which economists have equipped themselves with a half-baked knowledge of mathematical traditions has led to an un-natural mathematical economics and a non-numerical economic theory.

Whether this trend will reverse itself of its own volition is very doubtful. But discerning scholars of mathematical philosophy - including front-ranking mathematical theorists like Ulam - have seriously speculated, in the last few years, that the trend in mathematics itself may force a change in its methodology and epistemology. If mathematical traditions themselves incorporate the ambiguities of structures that are replete with undecidabilites in their bread-and-butter research, it will only be a matter of time before such habits will rub off on even the obdurate mathematical economist. Petty, our founding father, wanted only to 'express [himself] in number, weight or measure'. They need only to be linked together by means of parametric Diophantine equations - as Luca Pacioli knew when he devised that lasting contribution to mercantile practice: double-entry bookkeeping.

To get our 'pluses' and 'minuses' ordered so as to balance the books we do not need anything more than parametric Diophantine equations. Through them we enter the weird and wonderful world of undecidabilities and uncomputabilities, which is why the economic world, in its macroeconomic and microeconomic incarnations, is full of financial ambiguities. To pretend that the economic world can exist without such ambiguities and, hence, occasional and systematic exploitation of logical loopholes by unethical and immoral practitioners of financial wizardry is dangerous. On the other hand, recognizing the intrinsic inevitability of such ambiguities may mean that we might happily, in a quantitative economics of the future, return to the Linnean fold, to classify and systematise, particular intractable accounting schemes in increasingly and morally transparent ways. 


\section{Endnotes}

1. Undecidability and incompleteness conceived, naturally, in their formal senses.

2. I am not concerned in this paper with arbitrary applications of computability or undecidability theorems in an otherwise unmodified economic theory. I have had my say on such approaches in other writings and the interested reader is referred to them for detailed analysis, evaluation and criticisms (cf., for example, Velupillai, 2000, 2002 and 2004).

3. The five main subsections were titled:

- What is Mathematics?

- What is Physics?

- The Role of Mathematics in Physical Theories.

- Is the Success of Physical Theories Truly Surprising?

- The Uniqueness of the Theories of Physics.

4. The one footnote in which intuitionism is mentioned was a reference to Hilbert's disdainful dismissal of it (Wigner, 1960, footnote 4).

5. I found it surprising that Wigner, in a Richard Courant Lecture, did not refer to Courant's own famous attempt at providing an answer to the seemingly simple question 'What is Mathematics?' with a whole book with that title (Courant and Robbins, 1958). Courant's answer was to show what mathematics is by describing, explaining and demonstrating what they actually do. That was, perhaps, not suitable for Wigner's aims in the lecture.

6. I have in mind Auden's poignant eulogy to Yeats:

"Mad Ireland hurt you into poetry,

Now Ireland has her madness and her weather still,

For poetry makes nothing happen: it survives..."

Auden: 'In Memory of W.B.Yeats' (italics added).

7. Dummett's question, and enlightened answer, is entirely consistent with Hardy's analogous question and equally felicitous answer - except that the latter aimed at characterizing the mathematician:

"A mathematician, like a painter or a poet, is a maker of patterns. If his patterns are more permanent than theirs, it is because they are made with ideas. A painter makes patterns with shapes and colours, a poet with words..... .

The mathematician's patterns, like the painter's or the poet's, must be beautiful; the ideas, like the colours or the words, must fit together in a harmonious way. ...

It may be very hard to define mathematical beauty ... but that does not prevent us from recognising one when we [see] it."

Hardy, 1967, pp. 84-5; bold emphasis added.

Hardy's mathematician, who is a 'maker of beautiful patterns', is exactly Dummett's 'constructor of complex deductive arguments'. Just as Dummett's 'complex deductive arguments' are arrived at by 'routes far from immediately obvious', the 'beauty' of the patterns devised by Hardy's mathematicians are 'hard to define'.

8. As David Ruelle perceptively observed in his 'Gibbs Lecture':

"We like to think of the discovery of mathematical structure as walking upon a path laid out be the Gods. But .... may be there is no path....."

Ruelle, 1988, p.266. 
9. Adapted from Nelson (199?; no exact date in the source). Anticipating the characterisation of a Mathematical Economist in the next section, ME in this dialogue refers to such a being.

10. I could, instead, proceed, at this point, by asking the analogous question: what is a number? or any related question, substituting, for 'function' and 'number', other basic 'ideas' that are the objects manipulated by deductive arguments to construct the patterns that pave the route. For reasons of convenience and familiarity, I shall confine my discussion to the object referred to as 'function'.

11. I add the name Dirichlet to the standard naming which justly credits Kuratowski with this definition, for historical reasons. It was Dirichlet who initiated this particular tradition, culminating in Kuratowski's 'function as a graph' definition. Dirichlet's definition, in terms of open, continuous, intervals, remains the touchstone, as one can see from the way the Bishop-style constructivists and the Brouwerian Intuitionists have, eventually, defined functions. My source for this 'historical reason' is Hobson, 1927, p.274:

'It thus appears that an adequate definition of a function for a continuous interval $(a, b)$ must take the form given to it by Dirichlet'. Hobson does not elaborate upon the meaning of 'adequate', but it certainly had nothing to do with 'performing a task'.

Of course, the motivation and criteria in the latter two approaches were quite different from those of Dirichlet and Kuratowski.

12. And set theory is only one of at least four sub-branches of mathematical logic; the others being: proof theory, recursion theory and model theory. Loosely speaking, but not entirely inaccurately, it is possible to associate one particular class of numbers with each of these sub-branches of logic: real numbers, constructive numbers, computable numbers and non-standard numbers, respectively. Analogously, each of these forms the subject matter of: real analysis, constructive analysis, computable analysis and non-standard analysis. Which of these numbers and, hence, which kind of analysis, is appropriate for economic analysis is almost never discussed in any form or forum of mathematical economics or mathematics in economics. It is taken for granted that real numbers and its handmaiden, real analysis, is the default domain. Why?

13. Although it may appear paradoxical, I am of the opinion that non-standard analysis should be placed squarely in the constructive tradition - at least from the point of view of practice. Ever since Leibniz chose a notation for the differential and integral calculus that was conducive to computation, a notation that has survived even in the quintessentially non-computational tradition of classical real analysis, the practice of non-standard analysis has remained firmly rooted in applicability from a computational point of view. Indeed, the first modern rejuvenation of the non-standard tradition in the late $50 \mathrm{~s}$ and early $60 \mathrm{~s}$, at the hands of Schmieden and Laugwitz (1958), had constructive underpinnings. I add the caveat 'modern' because Veronese's sterling efforts (Veronese, 1891) at the turn of the 19th century did not succeed in revitalising the subject due to its unfair dismissal by Peano and Russell, from different points of view. The former dismissed it, explicitly, for lacking in 'rigour'; the latter, implicitly, by claiming that the triple problems of the infinitesimal, infinity and the continuum had been 'solved'.

14. Jacob Schwartz observed pungently:

"The very fact that a theory appears in mathematical form, that, for instance, a theory has provided the occasion for the application of a fixed-point theorem ... somehow makes us more ready to take it seriously. ... The result, perhaps most common in the social sciences, is bad theory with a mathematical passport. ... The intellectual attractiveness of a mathematical argument, ...., makes mathematics a powerful tool of intellectual prestidigitation - a glittering deception in which some are entrapped, and some, alas, entrappers."

Schwartz, 1986, pp. 22-3, italics added. 
15. Robert Kalaba was present at a seminar I gave in the department of economics at the University of Southern California, at the invitation of Richard Day, in the early 90s. I posed a similar question at that seminar and he enlightened the audience with the interesting story that, at Rand in Santa Monica, in the early $50 \mathrm{~s}$, intense discussion took place on suitable mathematical structures for economic formalizations and it was not, at that time, a foregone conclusion that real analysis and some variety of formalism - particularly the Bourbakian version - would come to dominate mathematical economics. A few years later Lloyd Shapley, during a luncheon conversation, on the occasion of a visit by Alain Lewis to the Center for Computable Economics in the department of economics at UCLA of which I was then the Associate Director, confirmed that such discussions did, indeed take place at Rand, particularly with Norman Shapiro. I have never been able to go beyond these anecdotal observations to substantiate a clear cut case one way or the other.

16. A question I have been framing, posing and asking mathematical economists, in alternative ways, both formally and informally, directly and indirectly, for many years without ever getting any satisfactory answer. It is not that the issue has not been addressed by eminent theorists in recent and less-than-recent years (Gomory and Baumol, 1960, Clower-Howitt, 1978 and Scarf, 1990). I shall return to this theme in the concluding section, too.

17. The paragraph and page references in the six 'bullet points' are all to Debreu (1959).

18. I should, for completeness, add a list of the deductive rules that are valid in different kids of mathematics, too. For example, the reason for the failure of the Bolzano-Weierstrass theorem in constructive mathematics is the uncritical use of the law of the excluded middle. This law and the law of double negation are the 'culprits' in the failure of the Brouwer fixed-point theorem in Brouwerian Intuitionistic mathematics. But I have refrained from making these explicit in view of the brief hints given in the previous section.

19. My translation of the last line from a stanza of one of Antonio Machado's great Cantares: "Caminante no hay camino, se hace camino al andar. Al andar se hace camino, y al volver la vista atras se ve la senda que nunca se ha de volver a pisar. ..."

20. It is impossible to justify or explain, within the brief span of a few paragraphs, the rich tapestry and the many-pronged structure of Wolfram's New Kind of Science. In the original version of this paper there was no mention of the relation of the message I am trying to convey in this paper and its possible embedding within Wolfram's vision. However, a perceptive referee expressed a wish to 'see some reference to Wolfram's New Kind of Science', pointing out, further, that NKS 'deals with some of the same areas as does this paper and might tie in the arguments better into broader work.' There is a great deal of truth in the referee's interesting remark and the few paragraphs do not do complete justice to the correctness of the conjecture. However, I do develop the relationship of the kind of message in this paper with Wolfram's vision in a separate paper (cf., Albin, Foley and Velupillai, 2004).

21. That there will be a role for Shapley Values and a place for cooperative game theory in such a vision is exemplified by the fact that cost accountants have, for years, grappled with the problem of joint cost imputations and joint production by using these concepts in fertile and imaginative ways. Shubik was early to recognise such a role for cooperative game theory and Shapley Values (cf. Shubik, 1962 and Shubik, 1986). There remains the problem that the domain of analysis for these concepts is the real number system. It will be an interesting challenge to device a formalism for cooperative game theory and its solutions in terms of computable or constructive numbers. 
22. The lasting contribution of economic analysis, to the mercantile culture of the modern era, was - in my opinion - double-entry bookkeeping. The Political Arithmetician and the Accountant has to deal with credit as well as the debit side of such bookkeeping discipline and, hence, it is not enough to confine attention to equations constrained by non-negative numbers. Negative numbers, even in their origin, play a role in double-entry bookkeeping.

23. Simply substitute 'economic theory' for 'probability theory', when reading this quote!

24. I have taken the liberty of substituting Diophantine equations for differential equations in the quoted paragraph.

25. Even knowledgeable scholars persist in referring to retroduction as abduction, in spite of Peirce explicitly stating: '.... $\alpha \pi \alpha \gamma \omega \gamma \eta$ should be translated not by the word abduction, as the custom of the translators is, but rather by reduction or retroduction'. (Peirce, 1898, p.141; italics in the original). 


\section{References}

Albin, P.S, Foley, D.K and Velupillai, K (2004), Review of A New Kind of Science by Stephen Wolfram (in preparation).

Arrow, K, J \& Hahn, F.H (1971), General Competitive Analysis, Holden-Day, Inc., San Francisco.

Beckett, S (1963), Watt, John Calder (Publishers) Ltd., London.

Brouwer, L. E. J (1952), An Intuitionist Correction of the Fixed-Point Theorem on the Sphere, Proceedings of the Royal Society: Series A, London, Vol. 213, June, pp. 1-2.

Burr, S. A, Editor, (1993), The Unreasonable Effectiveness of Number Theory, Proceedings of Symposia in Applied Mathematics, American Mathematical Society, Providence, RI.

Clower, R. W and Howitt, P.W (1978), The Transactions Theory of the Demand for Money: A Reconsideration, Journal of Political Economy, Vol. 86, no.3, pp. 449-466.

Cooper, N. G, Editor (1989), From Cardinals to Chaos - Reflections on the Life and Legacy of Stanislaw Ulam, Cambridge University Press, Cambridge.

da Costa, N. C.A and Doria, F.A (1994), Gödel Incompleteness in Analysis, with an Application to the Forecasting Problem in the Social Sciences, Philosophia Naturalis, Vol. 31, pp. 1-24.

Courant, R and Robbins, H (1958), What is Mathematics?, Oxford University Press, New York.

Debreu, G (1960), Theory of Value - An Axiomatic Analysis of Economic Equilibrium, John Wiley \& Sons, Inc., London.

Dummett, M (1977), Elements of Intuitionism, Clarendon Press, Oxford.

Dummett, M (1994), What is Mathematics About?, in: Mathematics and Mind edited by A. George, Chapter 1, pp. 11-26, Oxford University Press, Oxford.

Gomory, R. E \& Baumol, W.J (1960), Integer Programming and Pricing, Econometrica, Vol. 28, no.3, July, pp. 521-550.

Hamming, R W (1980), The Unreasonable Effectiveness of Mathematics, American Mathematical Monthly, Vol. 87, Issue 2, February, pp. 81-90.

Hamming, R. W (1991), The Art of Probability - For Scientists and Engineers, Addison-Wesley Publishing Company, Inc. Redwood City, California.

Hardy, G. H (1967), A Mathematician's Apology, Cambridge University Press, Cambridge.

Hicks, J. R (1956, [1982]), Methods of Dynamic Analysis, in: Money, Interest \& Wages - Collected Essays in Economic Theory, Volume II, Chapter 18, pp. 217-35.

Hicks, J. R (1983), A Discipline Not a Science, in: Classics and Moderns - Collected Essays on Economic Theory, Volume III, Chapter 32, pp. 365-75, Basil Blackwell, Oxford.

Hobson, E. W (1927), The Theory of Functions of a Real Variable \& the Theory of Fourier Series, Vol. 1, Third Edition, Cambridge University Press, Cambridge.

Keynes, J. M (1930, [1963]), Economic Possibilities for Our Grandchildren, in: Essays in Persuasion by John Maynard Keynes, Part V, Chapter 2, pp. 358-73, W.W. Norton \& Company, Inc., New York.

Kurz, H. D \& Salvadori, N (2001), Sraffa and the Mathematicians: Frank Ramsey and Alister Watson, in: Piero Sraffa's Political Economy-A Centenary Estimate, edited by Terenzio Cozzi \& Roberto Marchionatti, Routledge, London

Lesk, A (2000), The Unreasonable Effectiveness of Mathematics in Molecular Biology, The Mathematical Intelligencer, Vol. 22, No. 2, pp. 28-36.

Lesk, A (2001), Compared to What?, The Mathematical Intelligencer, Vol. 23, No. 1, p.4.

Matiyasevich, Y. V (1993), Hilbert's Tenth Problem, The MIT Press, Cambridge, Massachusetts.

McCauley, J. L, (2004), Dynamics of Markets: Econophysics and Finance, Cambridge University Press, Cambridge.

Metakides, G and Nerode, A (1982), The Introduction of Non-Recursive Methods into Mathematics, in: The L.E.J. Brouwer Centenary Symposium edited by A.S. Troelstra and D van Dalen, NorthHolland, Amsterdam.

Moschovakis, Y. N (1994), Notes on Set Theory, Springer-Verlag, Berlin. 
Nelson, E (199?), Understanding Intuitionism, Manuscript downloaded from: http://www.math.princeton.edu/ nelson/papers.html

Peirce, C. S (1898, [1992]), Reasoning and the Logic of Things - The Cambridge Conferences Lectures of 1898 edited by Kenneth Laine Ketner, with an Introduction by Kenneth Laine Ketner and Hilary Putnam, Harvard University Press, Cambridge, Massachusetts.

Polanyi, M (1958), Personal Knowledge: Towards a Post-Critical Philosophy, The University of Chicago Press, Chicago.

Prasad, K (2002), Variation and Observation in Finite Games, Manuscript, Department of Economics, Florida State University, May.

Ruelle, D (1988), Is Our Mathematics Natural? The Case of Equilibrium Statistical Mechanics, Bulletin (New Series) of the American Mathematical Society, Vol. 19, No. 1, July, pp. 259-68.

Sargent, T. J (1987), Macroeconomic Theory, Second Edition, Academic Press, Inc., London.

Scarf, H (1990), Mathematical Programming and Economic Theory, Operations Research, Vol. 38, pp. 377-85.

Schmieden, C \& Laugwitz, D (1958), Eine Erweiterung der Infinitesimalrechnung, Mathematisches Zeitschrift, Vol. 69, pp. 1-39.

Schwartz, J. T (1961), Lectures on the Mathematical Method in Analytical Economics, Gordon and Breach, Science Publishers, Inc., New York.

Schwartz, J. T (1986), The Pernicious Influence of Mathematics on Science, in: Discrete Thoughts Essays on Mathematics, Science, and Philosophy by Mark Kac, Gian-Carlo Rota and Jacob T. Schwartz, Birkhaüser, Boston.

Shubik, M (1962), Incentives, Decentralized Control, The Assignment of Joint Costs and Internal Pricing, Management Science, Vol. 8, no. 3, April, pp. 325-43.

Shubik, M (1986), The Cooperative Form, The Value, and the Allocation of Joint Costs and Benefits, in: Cost Allocation - Methods, Principles, Applications edited by H. Peyton Young, Chapter 4, pp. 79-94; Elsevier Science Publishers, Amsterdam.

Smale, S (1976), Dynamics in General Equilibrium Theory, American Economic Review, Vol. 66, No.2, May, pp.288-94.

Sraffa, P (1960), Production of Commodities by Means of Commodities: A Prelude to a Critique of Economic Theory, Cambridge University Press, Cambridge.

Temple, G (1958), Linearization and Delinearization, Proceedings of the International Congress of Mathematicians, pp. 233-47, Cambridge University Press, Cambridge.

Tsuji, M, da Costa, N.C.A and Doria, F.A (1998), 'The Incompleteness Theories of Games', Journal of Philosophical Logic, Vol.m27, pp.553-68.

Uzawa, H (1962), 'Walras' Existence Theorem and Brouwer's Fixed-Point Theorem', The Economic Studies Quarterly, Vol. 8, No.1, pp.59-62.

Velupillai, K (1989), The Existence of the Standard System: Sraffa's Constructive Proof, Political Economy - Studies in the Surplus Approach, Vol. 5, no.1, pp.3-12.

Velupillai, K (2000), Computable Economics, Oxford University Press, Oxford.

Velupillai, K (2002), Effectivity and Constructivity in Economic Theory, Journal of Economic Behavior \& Organization, Vol. 49, no. 3, November, pp. 307-25.

Velupillai, K (2004), Rational Expectations Equilibria: A Recursion Theoretic Tutorial, in: Macroeconomic Theory and Economic Policy - Essays in Honour of Jean-Paul Fitoussi edited by K.Velupillai, Chapter 11, pp.169-88; Routledge, London.

Veronese, G (1891), Fondamenti di geometria a più dimensioni e a più specie di unità rettilinee espositi in forma elementare, Tipografia del Seminario, Padova.

Wigner, E (1960), 'The Unreasonable Effectiveness of Mathematics in the Natural Sciences', Communications in Pure and Applied Mathematics, Vol. 13, pp. 1-14.

Wigner, E (1964), 'Events, Laws of Nature and Invariance Principles', Science, Vol. 145, no. 3636, 4 September, pp. 995-9. 
Wolfram, S (2002), A New Kind of Science, Wolfram Media, Inc., Champaign, IL. 
Elenco dei papers del Dipartimento di Economia

2000.1 A two-sector model of the effects of wage compression on unemployment and industry distribution of employment, by Luigi Bonatti

2000.2 From Kuwait to Kosovo: What have we learned? Reflections on globalization and peace, by Roberto Tamborini

2000.3 Metodo e valutazione in economia. Dall'apriorismo a Friedman, by Matteo Motterlini

2000.4 Under tertiarisation and unemployment. by Maurizio Pugno

2001.1 Growth and Monetary Rules in a Model with Competitive Labor Markets, by Luigi Bonatti.

2001.2 Profit Versus Non-Profit Firms in the Service Sector: an Analysis of the Employment and Welfare Implications, by Luigi Bonatti, Carlo Borzaga and Luigi Mittone.

2001.3 Statistical Economic Approach to Mixed Stock-Flows Dynamic Models in Macroeconomics, by Bernardo Maggi and Giuseppe Espa.

2001.4 The monetary transmission mechanism in Italy: The credit channel and a missing ring, by Riccardo Fiorentini and Roberto Tamborini.

2001.5 Vat evasion: an experimental approach, by Luigi Mittone

2001.6 Decomposability and Modularity of Economic Interactions, by Luigi Marengo, Corrado Pasquali and Marco Valente.

2001.7 Unbalanced Growth and Women's Homework, by Maurizio Pugno

2002.1 The Underground Economy and the Underdevelopment Trap, by Maria Rosaria Carillo and Maurizio Pugno.

2002.2 Interregional Income Redistribution and Convergence in a Model with Perfect Capital Mobility and Unionized Labor Markets, by Luigi Bonatti.

2002.3 Firms' bankruptcy and turnover in a macroeconomy, by Marco Bee, Giuseppe Espa and Roberto Tamborini.

2002.4 One "monetary giant" with many "fiscal dwarfs": the efficiency of macroeconomic stabilization policies in the European Monetary Union, by Roberto Tamborini. 
2002.5 The Boom that never was? Latin American Loans in London 1822-1825, by Giorgio Fodor.

2002.6 L'economia senza banditore di Axel Leijonhufoud: le 'forze oscure del tempo e dell'ignoranza' e la complessità del coordinamento, by Elisabetta De Antoni.

2002.7 Why is Trade between the European Union and the Transition Economies Vertical?, by Hubert Gabrisch and Maria Luigia Segnana.

2003.1 The service paradox and endogenous economic gorwth, by Maurizio Pugno.

2003.2 Mappe di probabilità di sito archeologico: un passo avanti, di Giuseppe Espa, Roberto Benedetti, Anna De Meo e Salvatore Espa.

(Probability maps of archaeological site location: one step beyond, by Giuseppe Espa, Roberto Benedetti, Anna De Meo and Salvatore Espa).

2003.3 The Long Swings in Economic Understianding, by Axel Leijonhufvud.

2003.4 Dinamica strutturale e occupazione nei servizi, di Giulia Felice.

2003.5 The Desirable Organizational Structure for Evolutionary Firms in Static Landscapes, by Nicolás Garrido.

2003.6 The Financial Markets and Wealth Effects on Consumption An Experimental Analysis, by Matteo Ploner.

2003.7 Essays on Computable Economics, Methodology and the Philosophy of Science, by Kumaraswamy Velupillai.

2003.8 Economics and the Complexity Vision: Chimerical Partners or Elysian Adventurers?, by Kumaraswamy Velupillai.

2003.9 Contratto d'area cooperativo contro il rischio sistemico di produzione in agricoltura, di Luciano Pilati e Vasco Boatto.

2003.10 Il contratto della docenza universitaria. Un problema multi-tasking, di Roberto Tamborini.

2004.1 Razionalità e motivazioni affettive: nuove idee dalla neurobiologia e psichiatria per la teoria economica? di Maurizio Pugno.

(Rationality and affective motivations: new ideas from neurobiology and psychiatry for economic theory? by Maurizio Pugno.

2004.2 The economic consequences of Mr. G. W. Bush's foreign policy. Can th US afford it? by Roberto Tamborini

2004.3 Fighting Poverty as a Worldwide Goal by Rubens Ricupero

2004.4 Commodity Prices and Debt Sustainability by Christopher L. Gilbert and Alexandra Tabova 
2004.5 A Primer on the Tools and Concepts of Computable Economics by K. Vela Velupillai

2004.6 The Unreasonable Ineffectiveness of Mathematics in Economics by K. Vela Velupillai 
PUBBLICAZIONE REGISTRATA PRESSO IL TRIBUNALE DI TRENTO 